\title{
Vision based aircraft tracking under LPC coordinate system
}

\author{
Nam Trung Pham, Jean-Pierre Le Cadre \\ IRISA/INRIA-CNRS, \\ Campus de Beaulieu, 35042 Rennes Cedex, France, \\ (e-mail: ntpham, lecadre@irisa.fr)
}

\begin{abstract}
Implementations of the Bayes filtering for vision based aircraft tracking suffer fundamental difficulties in the Cartesian coordinate. This is because of the uncertainty of the noise model in the flight dynamic equation when the aircraft state is described in these coordinates. Recently, the Logarithmic Polar Coordinate (LPC) framework has been successfully applied in the bearings-only tracking context, with the ability of estimating the variance to range ratio (Brehard and Le Cadre [2004]). In this paper, we design an aircraft tracking method using LPC. This method can adapt with changes of the noise model in the flight dynamic equation. Hence, it is more robust than methods for vision based aircraft tracking using the Cartesian Coordinates.
\end{abstract}

Keywords: Target tracking, tracking filters, aircraft control, navigation, Monte Carlo method

\section{INTRODUCTION}

This paper addresses the problem of estimating the position and velocity of an aircraft by using projections of corner points of the runway in the image plane. The camera is attached to the aircraft. When the aircraft moves, information from the projections of interest points will help to estimate the aircraft state. These interest points can be detected by runway tracking or runway detection that can be done by using image processing methods (Hough transform, active contour (Barat and Lagadec [2008]), segmentation methods (Kolmogorov and Zabih [2004]), etc.). This is an important task for automatic landing system, or navigation system.

Vision based aircraft tracking has been considered in several papers (Dobrokhodov et al. [2006], Stepanyan and Hovakimyan [2008], Bryson and Sukkarieh [2005], Tisdale et al. [2008], Kaminer et al. [2001], Hespanha et al. [2004]). Most of them defined a flight dynamic model and a measurement model. The flight dynamic is corrupted by a process noise. In (Hespanha et al. [2004], Kaminer et al. [1999], and Kaminer et al. [2001]), filtering methods are designed for aircraft tracking. They applied the linear parametrically varying system theory to prove the regional stability in the performance when the time goes to infinity. Based on these works, Dobrokhodov et al. [2006] included the loss target tracking event in the system model to have a more robust system. Some other methods rely on the extended Kalman filter, the unscented Kalman filter, or the particle filter to obtain the aircraft state estimation (Bryson and Sukkarieh [2005], Tisdale et al. [2008]). Bryson and Sukkarieh [2005] assume that the feature detection method can find multiple points of interests in the image. So, the observability of the aircraft state in this method is higher than other methods (Hespanha et al. [2004], Kaminer et al. [1999], and Kaminer et al. [2001]) that assume only one interest point is available. When the aircraft is very far from interest points or the number of interest points is small, the observability of the aircraft state is reduced. This will affect the tracking performance. Moreover, when the aircraft moves, parameters in the state space model can be changed with the time, for example the noise in the flight model. These problems enhance the uncertainty in the estimation and cause challenges for aircraft tracking.

Rather than using Cartesian coordinate in tracking, the polar coordinate is applied successfully in bearings-only tracking (Aidala and Hammel [1983], Allen and Blackman [1991], Karlsson and Gustafsson [2001], Brehard and Le Cadre [2004], Brehard and Le Cadre [2007]). It makes the tracking method be more robust because of taking into account the poor observability of the radial distance. Especially, in (Brehard and Le Cadre [2004], Brehard and Le Cadre [2007]), the variance to range ratio is proved to be observable under the modified polar coordinate or logarithm polar coordinate. So, the uncertainty of the noise model in the state equation can be reduced during the tracking period.

In this paper, we apply LPC for vision based aircraft tracking. An LPC state space model for the 3D aircraft tracking system is derived. Then the particle filter (Arulampalam et al. [2002]) with LPC state space is used to obtain the aircraft state. Describing the aircraft state in LPC results in a dramatic improvement of the tracking performance.

Section 2 provides formulations of the problem in the Cartesian coordinate and describes the aircraft tracking system. Section 3 details formulations of LPC state space in 3D. Section 4 describes the particle filter algorithm for aircraft tracking. Finally, section 5 and 6 show our results, comparisons, and conclusions. 


\section{PROBLEM FORMULATIONS IN CARTESIAN COORDINATE}

The aircraft is equipped with a camera. The inertial reference frame $\{G\}$ is described in the north east down (NED) coordinate. Let $\{B\}$ be the aircraft body frame, and $\{C\}$ be the camera frame. The aircraft state in $\{G\}$ at time $k$ is described by $X_{k}=\left\{x_{k}, y_{k}, z_{k}, \dot{x}_{k}, \dot{y}_{k}, \dot{z}_{k}\right\}$. It is the position and velocity of aircraft at time $k$ in $\{G\}$. We assume that the aircraft moves with the following flight dynamic equation

$$
X_{k}=F_{k-1} X_{k-1}+\sigma w_{k-1}
$$

where $w_{k-1} \sim \mathcal{N}(0, Q)$ is the process noise,

$$
F_{k-1}=\left[\begin{array}{cc}
I d_{3} & \eta_{k-1} I d_{3} \\
0 & I d_{3}
\end{array}\right]
$$

where $\eta_{k-1}$ is the time interval between $k-1$ and $k, I d_{n}$ is the $n \times n$ identity matrix. There are some assumptions:

i. Euler angles $\left\{\phi_{B}^{C}, \theta_{B}^{C}, \psi_{B}^{C}\right\}$ and the translation $\left\{t_{B}^{C}\right\}$ between camera and the aircraft are known.

ii. Aircraft Euler angles $\left\{\phi_{G}^{B}, \theta_{G}^{B}, \psi_{G}^{B}\right\}$ and the altitude of the aircraft are known.

The coordinate system is presented in Figure 1. The definitions of Euler angles $\left\{\phi_{G}^{B}, \theta_{G}^{B}, \psi_{G}^{B}\right\}$ are shown in Figure 2 (it is similar for Euler angles $\left\{\phi_{B}^{C}, \theta_{B}^{C}, \psi_{B}^{C}\right\}$ ). They are rotation angles with $x, y, z$ axis, respectively. The order of rotations is as follows: first, rotate around $z$-axis by the angle $\psi_{G}^{B}$, then rotate around the new $y$-axis by angle $\theta_{G}^{B}$, and finally rotate around the newest $x$-axis by angle $\phi_{G}^{B}$. Assumption (i) is easy to understand because we can setup these parameters. Assumption (ii) can be obtained by attitude estimation instruments and pressure altitude sensors (Hespanha et al. [2004]).

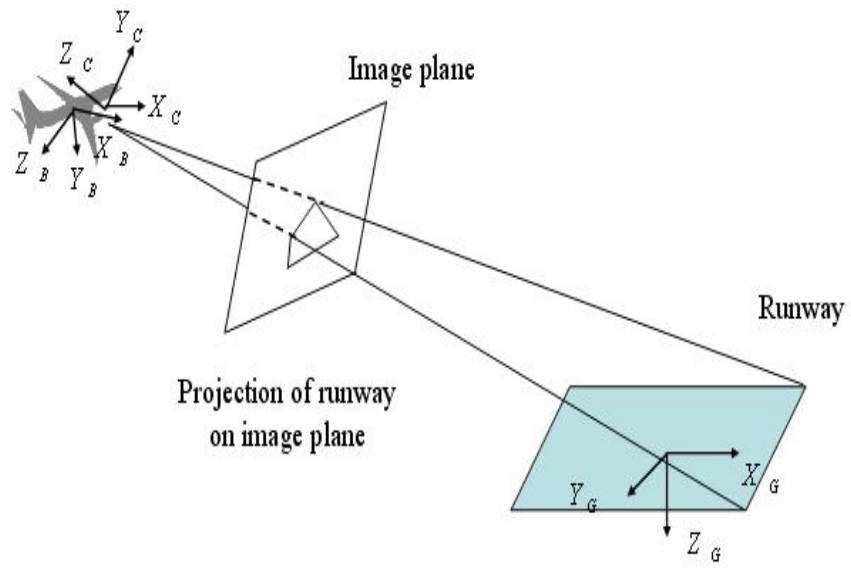

Fig. 1. Coordinate system

When the aircraft moves, the camera captures images from the runway area. Some of methods to detect and track the runway with image data are performed to obtain the projections of corner points of the runway. One possible method is to detect the runway by using Hough transform and track the runway by segmentation or energy minimization methods (Kolmogorov and Zabih [2004], Barat and

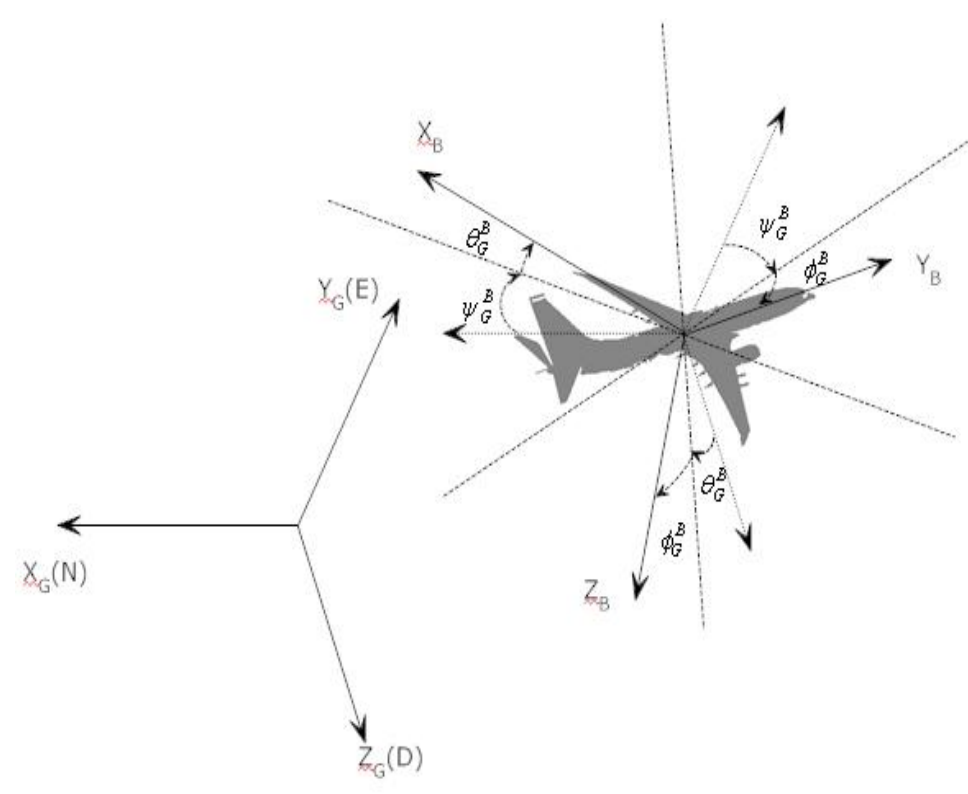

Fig. 2. Euler angles to transform from $\{\mathrm{G}\}$ to $\{\mathrm{B}\}$

Lagadec [2008]). Figure 3 shows the detected runway by red lines. Hence, we can have corner points of the runway in the image plane.

Let $M_{B}^{C}=M\left(\phi_{B}^{C}, \theta_{B}^{C}, \psi_{B}^{C}, t_{B}^{C}\right)$ be the homogeneous transformation matrix with Euler angles $\left\{\phi_{B}^{C}, \theta_{B}^{C}, \psi_{B}^{C}\right\}$ and translation $t_{B}^{C}$ from aircraft body to camera, and $M_{G}^{B}\left(X_{k}^{1: 3}\right)=M\left(\phi_{G}^{B}, \theta_{G}^{B}, \psi_{G}^{B}, X_{k}^{1: 3}\right)$ be the homogeneous transformation matrix with Euler angles $\left\{\phi_{G}^{B}, \theta_{G}^{B}, \psi_{G}^{B}\right\}$ and translation $X_{k}^{1: 3}$ (aircraft position $X_{k}^{1: 3}=\left\{x_{k}, y_{k}, z_{k}\right\}$ ) from $\{G\}$ to $\{B\}$. Let $Z_{k}=\left\{z_{k}^{1}, . ., z_{k}^{4}, z_{k}^{5}\right\}$, where $\left\{z_{k}^{1}, . ., z_{k}^{4}\right\}$ are the positions of the four corner points of the runway in the image and $z_{k}^{5}$ is the altitude measurement. The measurement equation is defined as follows. First, we consider the $i$-th corner point $P_{i}$. The projection from $P_{i}$ in $\{G\}$ to $\{C\}$ is

$$
\left[\begin{array}{c}
x_{c} \\
y_{c} \\
z_{c} \\
1
\end{array}\right]=M_{B}^{C} M_{G}^{B}\left(X_{k}^{1: 3}\right) P_{i}
$$

Then,

$$
z_{k}^{i}=\left[\begin{array}{l}
u_{0}+f \frac{x_{c}}{z_{c}} \\
v_{0}+f \frac{y_{c}}{z_{c}}
\end{array}\right]+v_{k}^{i}
$$

where $\left(u_{0}, v_{0}\right)$ is the camera principal point, $f$ is the focal length, and $v_{k}^{i}$ is the measurement noise for observation $z_{k}^{i}, v_{k}^{i} \sim \mathcal{N}(0, R)$. The altitude measurement equation is

$$
z_{k}^{5}=X_{k}^{3}+v_{k}^{5}
$$

Therefore, the measurement equation can be written as follows

$$
Z_{k}=h\left(X_{k}\right)+v_{k}
$$

Let $Z_{1: k}=\left\{Z_{1}, Z_{2}, \ldots, Z_{k}\right\}$ be the observations up to time $k$. The aim of the Bayes filter is to estimate the posterior density $p\left(X_{k} \mid Z_{1: k}\right)$. Some implementations of 
the Bayes filter can be applied in this case such as extended Kalman filter, unscented Kalman filter, or particle filter (Arulampalam et al. [2002]).

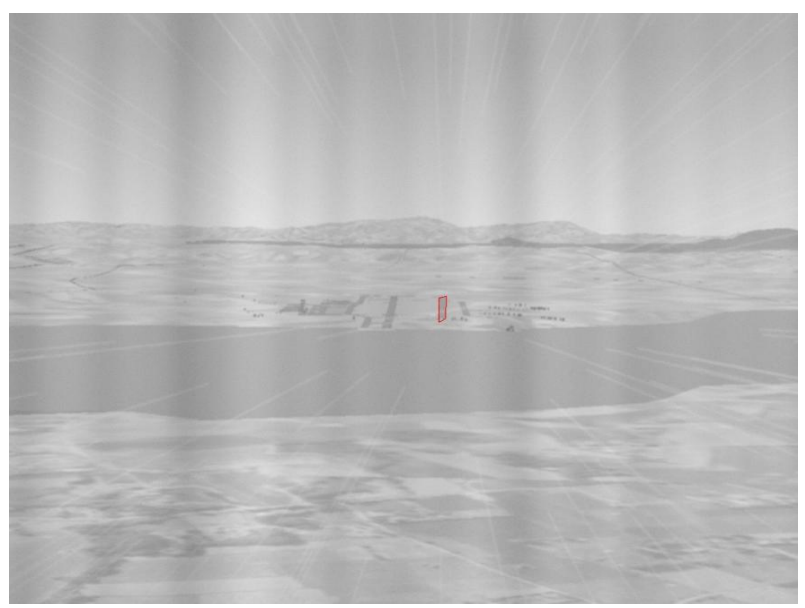

Fig. 3. Runway detection

\section{LPC FOR 3D AIRCRAFT STATE SPACE MODEL}

The variance $\sigma$ of the noise model in equation (1) is usually not known in applications. Therefore, we usually use the $\sigma_{\max }$ value to deal with changes of aircraft motion modes. This may affect the accuracy of the estimation. Fortunately, Brehard and Le Cadre [2004] shows that the variance to range ratio can also be estimated within the LPC framework. Now, we extend this work to the 3D case and apply it in the aircraft tracking.

The aircraft state vector in LPC is defined as follows

$$
Y_{k}=\left[\alpha_{k}, \beta_{k}, \rho_{k}, \dot{\alpha}_{k}, \dot{\beta}_{k}, \dot{\rho}_{k}, \tilde{\sigma}_{k}\right]
$$

where $\alpha_{k}$ is the angle from the $x$-axis, $\beta_{k}$ is the angle from the $z$-axis, $\rho_{k}$ is the logarithm of the distance from origin to the target, and $\tilde{\sigma}_{k}=\frac{\sigma}{r_{k}}$ is the variance to range ratio, $r_{k}$ is the distance from target to origin in $\{G\}$. Figure 4 shows more details about $\alpha_{k}, \beta_{k}$, and $\rho_{k}$.

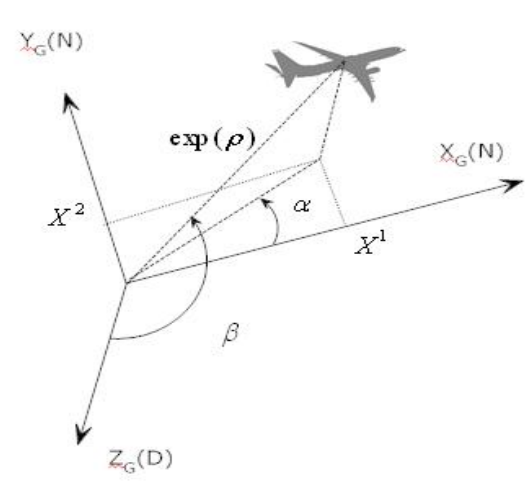

Fig. 4. Definition of logarithmic polar coordinate terms: $\alpha$,

$$
\beta, \rho
$$

Let

$$
\mathcal{R}_{k}=\left[\begin{array}{ccc}
\sin \beta_{k} \cos \alpha_{k} & \cos \beta_{k} \cos \alpha_{k} & -\sin \beta_{k} \sin \alpha_{k} \\
\sin \beta_{k} \sin \alpha_{k} & \cos \beta_{k} \sin \alpha_{k} & \sin \beta_{k} \cos \alpha_{k} \\
\cos \beta_{k} & -\sin \beta_{k} & 0
\end{array}\right]
$$

We have the relation of the aircraft velocity in Cartesian coordinate and LPC as follows

$$
\left[\begin{array}{c}
\dot{x}_{k} \\
\dot{y}_{k} \\
\dot{z}_{k}
\end{array}\right]=\mathcal{R}_{k}\left[\begin{array}{c}
r_{k} \dot{\rho}_{k} \\
r_{k} \dot{\beta}_{k} \\
r_{k} \dot{\alpha}_{k}
\end{array}\right]
$$

Let $f_{L P C}^{C}$ be the transformation function from LPC to Cartesian coordinate.

$$
\begin{aligned}
X_{k} & =f_{L P C}^{C}\left(Y_{k}^{1: 6}\right) \\
& =r_{k}\left[\begin{array}{cc}
\mathcal{R}_{k} & 0 \\
0 & \mathcal{R}_{k}
\end{array}\right]\left[\begin{array}{c}
1 \\
0 \\
0 \\
\dot{\rho}_{k} \\
\dot{\beta}_{k} \\
\dot{\alpha}_{k}
\end{array}\right]
\end{aligned}
$$

From (1), the flight dynamic equation can be written as follows

$$
\begin{aligned}
& X_{k}=F_{k-1} X_{k-1}+\sigma w_{k-1} \\
& =F_{k-1} r_{k-1}\left[\begin{array}{cc}
\mathcal{R}_{k-1} & 0 \\
0 & \mathcal{R}_{k-1}
\end{array}\right]\left[\begin{array}{c}
1 \\
0 \\
0 \\
\dot{\rho}_{k-1} \\
\dot{\beta}_{k-1} \\
\dot{\alpha}_{k-1}
\end{array}\right]+\sigma w_{k-1} \\
& =r_{k-1}\left[\begin{array}{cc}
I d_{3} & \eta_{k-1} I d_{3} \\
0 & I d_{3}
\end{array}\right]\left[\begin{array}{cc}
\mathcal{R}_{k-1} & 0 \\
0 & \mathcal{R}_{k-1}
\end{array}\right]\left[\begin{array}{c}
1 \\
0 \\
0 \\
\dot{\rho}_{k-1} \\
\dot{\beta}_{k-1} \\
\dot{\alpha}_{k-1}
\end{array}\right]+\sigma w_{k-1} \\
& =r_{k-1}\left[\left[\begin{array}{cc}
1 & \eta_{k-1} \\
0 & 1
\end{array}\right] \otimes I d_{3}\right]\left[I d_{2} \otimes \mathcal{R}_{k-1}\right]\left[\begin{array}{c}
1 \\
0 \\
0 \\
\dot{\rho}_{k-1} \\
\dot{\beta}_{k-1} \\
\dot{\alpha}_{k-1}
\end{array}\right]+\sigma w_{k-1} \\
& =r_{k-1}\left[I d_{2} \otimes \mathcal{R}_{k-1}\right]\left[\begin{array}{c}
1+\eta_{k-1} \dot{\rho}_{k-1} \\
\eta_{k-1} \dot{\beta}_{k-1} \\
\eta_{k-1} \dot{\alpha}_{k-1} \\
\dot{\rho}_{k-1} \\
\dot{\beta}_{k-1} \\
\dot{\alpha}_{k-1}
\end{array}\right]+\sigma w_{k-1} \\
& =r_{k-1}\left[\begin{array}{c}
V_{1} \\
V_{2} \\
V_{3} \\
V_{4} \\
V_{5} \\
V_{6}
\end{array}\right]
\end{aligned}
$$

where

$$
\left[\begin{array}{c}
V_{1} \\
V_{2} \\
V_{3} \\
V_{4} \\
V_{5} \\
V_{6}
\end{array}\right]=\left[I d_{2} \otimes \mathcal{R}_{k-1}\right]\left[\begin{array}{c}
1+\eta_{k-1} \dot{\rho}_{k-1} \\
\eta_{k-1} \dot{\beta}_{k-1} \\
\eta_{k-1} \dot{\alpha}_{k-1} \\
\dot{\rho}_{k-1} \\
\dot{\beta}_{k-1} \\
\dot{\alpha}_{k-1}
\end{array}\right]+\frac{\sigma}{r_{k-1}} w_{k-1}
$$


Let $f_{C}^{L P C}$ be the transformation function from Cartesian to LPC.

$$
\begin{aligned}
& Y_{k}^{1: 6}= f_{C}^{L P C}\left(X_{k}\right) \\
&= {\left[\begin{array}{c}
S_{1, k} \\
S_{2, k} \\
\ln \left(\sqrt{\left(X_{k}^{1}\right)^{2}+\left(X_{k}^{2}\right)^{2}+\left(X_{k}^{3}\right)^{2}}\right) \\
\dot{S}_{1, k} \\
\dot{S}_{2, k} \\
\frac{\partial}{\partial t}\left(\begin{array}{c}
\ln \left(\sqrt{\left(X_{k}^{1}\right)^{2}+\left(X_{k}^{2}\right)^{2}+\left(X_{k}^{3}\right)^{2}}\right)
\end{array}\right) \\
S_{1, k} \\
S_{2, k} \\
\left.\sqrt{V_{1}^{2}+V_{2}^{2}+V_{3}^{2}}\right) \\
\frac{V_{1} V_{5}-V_{2} V_{4}}{V_{1}^{2}+V_{2}^{2}} \\
\rho_{k-1}+\ln \left(\begin{array}{c}
2 \\
V_{1} V_{3} V_{4}+V_{2} V_{3} V_{5}-V_{1}^{2} V_{6}-V_{2}^{2} V_{6} \\
\left(V_{1}^{2}+V_{2}^{2}+V_{3}^{2}\right) \sqrt{V_{1}^{2}+V_{2}^{2}} \\
\frac{V_{1} V_{4}+V_{2} V_{5}+V_{3} V_{6}}{\left(V_{1}^{2}+V_{2}^{2}+V_{3}^{2}\right)}
\end{array}\right]
\end{array}\right.} \\
&
\end{aligned}
$$

where

$$
\begin{gathered}
S_{1}=\operatorname{atan} 2\left(V_{2}, V_{1}\right) \\
S_{2}=\operatorname{atan} 2\left(\sqrt{V_{1}^{2}+V_{2}^{2}}, V_{3}\right)
\end{gathered}
$$

Moreover, the distance $r_{k}$ is obtained by

$$
\begin{aligned}
r_{k} & =\sqrt{\left(X_{k}^{1}\right)^{2}+\left(X_{k}^{2}\right)^{2}+\left(X_{k}^{3}\right)^{2}} \\
& =r_{k-1} \sqrt{V_{1}^{2}+V_{2}^{2}+V_{3}^{2}}
\end{aligned}
$$

So,

$$
Y_{k}^{7}=\frac{Y_{k-1}^{7}}{\sqrt{V_{1}^{2}+V_{2}^{2}+V_{3}^{2}}}
$$

From equations (12) and (16), the aircraft state equation in LPC can be written as follows

$$
Y_{k}=f_{1}\left(Y_{k-1}, w_{k-1}\right)
$$

Furthermore, we know that the transformation of $X_{k}^{1: 3}$ to $\mathrm{LPC}$ is

$$
X_{k}^{1: 3}=\left[\begin{array}{c}
\exp \left(\rho_{k}\right) \sin \beta_{k} \cos \alpha_{k} \\
\exp \left(\rho_{k}\right) \sin \beta_{k} \sin \alpha_{k} \\
\exp \left(\rho_{k}\right) \cos \beta_{k}
\end{array}\right]
$$

Therefore, equation (6) can be written as

$$
Z_{k}=h_{1}\left(Y_{k}\right)+v_{k} \text {. }
$$

With the definitions in equations (17) and (19), the state space model is transformed from Cartesian coordinate to LPC. In $\mathrm{LPC}$, the variance to range ratio $\tilde{\sigma}_{k}$ can be estimated because of the proof in (Brehard and Le Cadre [2004]). Hence, the noise in the flight model under LPC can be adapted with the moving of aircraft. This is the key to develop a robust method for vision based aircraft tracking.

\section{PARTICLE FILTER FOR AIRCRAFT TRACKING}

We apply the particle filter (Arulampalam et al. [2002]) to track the aircraft. First, assuming that the density at the beginning $p\left(X_{0}\right)$ is represented by a set of samples $\left\{X_{0}^{(i)}\right\}_{i=1}^{N}$ and the variance of $p\left(X_{0}\right)$ is $\sigma_{0}$. Then, we use equation (12) and $\sigma_{0}$ to create the initialization sample set in $\operatorname{LPC}\left\{Y_{0}^{(i)}\right\}_{i=1}^{N}$, where

$$
Y_{0}^{7(i)}=\tilde{\sigma}_{0}^{(i)} \sim \frac{\sigma_{0}}{r_{0}^{(i)}} \mathcal{U}(0,1)
$$

where $\mathcal{U}(0,1)$ is the uniform distribution in $[0,1]$. At each time step $k$, samples at time $k-1,\left\{Y_{k-1}^{(i)}\right\}_{i=1}^{N}$, will be used to predict samples at time $k,\left\{\tilde{Y}_{k}^{(i)}\right\}_{i=1}^{N}$, by using equation (17). These samples are hypothesized aircraft states. Then, runway corner points are projected on the image plane by using hypothesized aircraft states to create a predicted measurement set. After that, the weight for each sample will be assigned based on the predicted measurement set and detected measurements in image. This stage is implemented by using equation (19). Sample weights are normalized and we can obtain a set of sample $\left\{Y_{k}^{(i)}\right\}_{i=1}^{N}$ represented for the updated distribution. Resampling methods can be applied to avoid sample degeneracy. Finally, the state of aircraft is estimated by the updated distribution. The details of the algorithm are described in Figure 5.

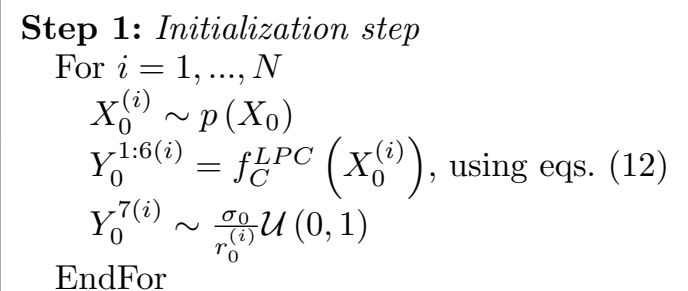

At time $k \geq 1$

Step 2: Sampling step

For $i=1, \ldots, N$

Prediction $\tilde{Y}_{k}^{(i)}=f_{1}\left(Y_{k-1}^{(i)}, w_{k-1}^{(i)}\right)$, eqs. (17)

Detect observation $Z_{k}$ in the image $I_{k}$

Weighting $\tilde{w}_{k}^{(i)}=g_{k}\left(Z_{k} \mid \tilde{Y}_{k}^{(i)}\right) w_{k-1}^{(i)}$, eqs. (19)

EndFor

Normalized weights: $\sum_{i=1}^{N} \tilde{w}_{k}^{(i)}=1$.

Step 3: Estimation step

Resample $\left\{\tilde{w}_{k}^{(i)}, \tilde{Y}_{k}^{(i)}\right\}_{i=1}^{N}$ to get $\left\{w_{k}^{(i)}, Y_{k}^{(i)}\right\}_{i=1}^{N}$

Estimate $\hat{Y}_{k}$, using eqs. (10)

to obtain aircraft state in $\{G\}$

Return step 2 for the next time $k+1$

Fig. 5. Particle filter for vision based aircraft tracking

\section{SIMULATION RESULTS}

The algorithm is tested under the Pegase simulation system. This simulation system is a study of a navigation sys- 
tem in Pegase project (http://dassault.ddo.net/pegase/) whose objectives are to prepare the development of an autonomous (no external assistance or ground equipment required) in all weather conditions, localization and guidance system based on vision sensor outputs. Some scenarios are considered such as low resolution image $(512 \times 640)$, high resolution image $(1024 \times 1280)$, frog, rain, etc. Some parameters for the system are described in Table 1.

\begin{tabular}{|l|l|}
\hline Parameters & Value \\
\hline \hline$\sigma_{0}$ & $100 \mathrm{~m}$ \\
\hline$N$ & 800 \\
\hline$\sigma_{v}^{p}$ & 2 \\
\hline$\sigma_{v}^{a}$ & 10 \\
\hline$\eta_{k}$ & $0.1 \mathrm{~s}$ \\
\hline
\end{tabular}

Table 1. Parameters for particle filter

First, we compare the LPC approach with the particle filter in Cartesian Coordinates (CC). The initialization position error is $[600,300,200]$. In this scenario, the aircraft is about $14000 \mathrm{~m}$ far from the runway. Figure 6 shows that the CC particle filter is diverging with time. This is because the initialization is not good, the number of particles is limited, and the flight model is not well defined for bad initializations. Hence, the particle filter in Cartesian coordinate cannot recover from a poor initialization. Opposite, we can see in Figure 6 that our method performs quite satisfactorily. The method can adapt well with changes of aircraft motion mode. The state estimates gradually converge to the true trajectory even it is badly initialized. This is because of the ability of estimating the variance to range ratio via $\mathrm{LPC}$.

Figure 7 shows the performance of our method in the whole tracking period. There are 2500 frames in about 5 minutes before the aircraft lands to the runway. The figure shows that the performance is quite stable throughout the whole experiment.

Figure 8 shows the comparison of mean square error (MSE) of our method and the particle filter in Cartesian with good initializations. The figure shows that the MSE of the particle filter in LPC is smaller and more stable than the $\mathrm{CC}$ one. In most of time, the MSE of the particle filter in LPC is close to the posterior Cramer round bound (PCRB). The high errors from time steps 1500 to 2000 are because of high errors in the measurement detection. In these time steps, there are some trees near the runway and they affect the performance of measurement detection; while the PCRB uses "ideal" measurements. However, LPC tracking is able to recover from these false measurements.

\section{CONCLUSION}

In this paper, we developed the LPC framework in vision based aircraft tracking. The algorithm is tested under the Pegase simulation system. This method largely outperforms any filter using the classical Cartesian coordinates. In $\mathrm{LPC}$, we can estimate the variance to range ratio. The noise model will change automatically according to the aircraft motion mode. This is the key for a good behavior of the tracking / navigation system. Moreover, this method
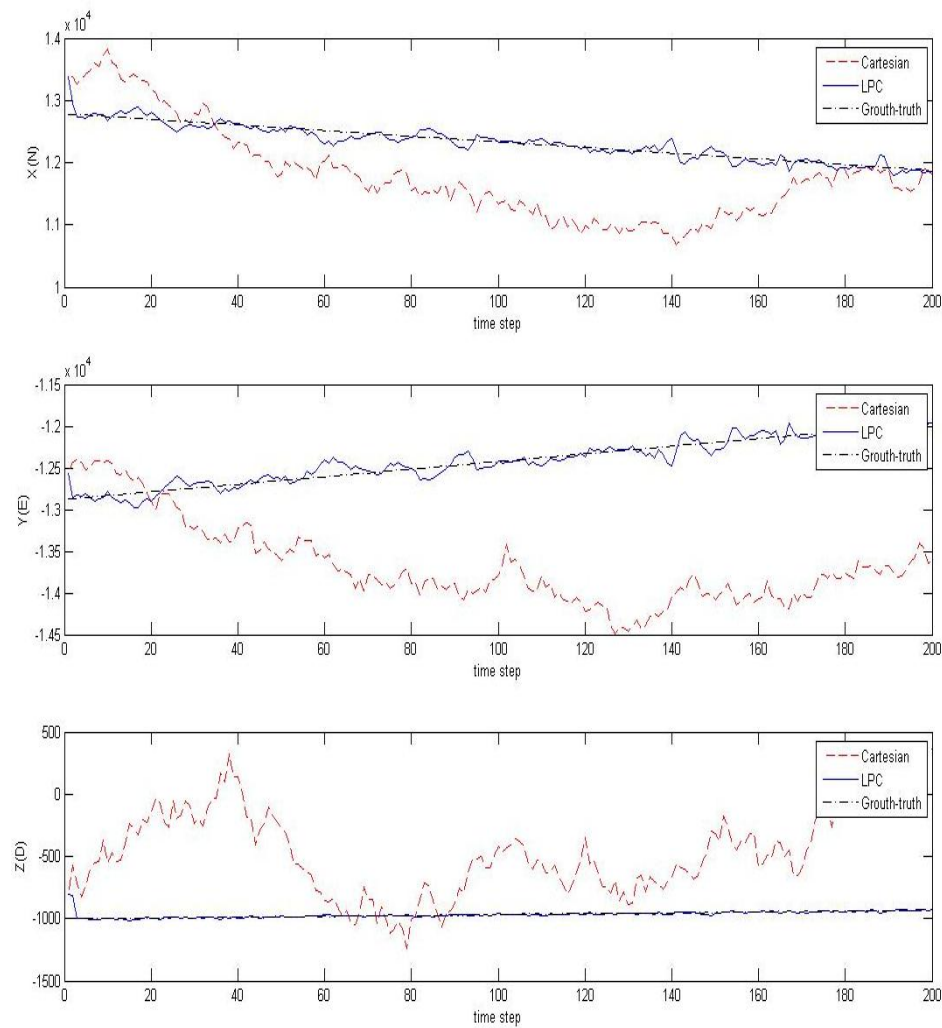

Fig. 6. Performance comparison between particle filter in LPC and Cartesian coordinate
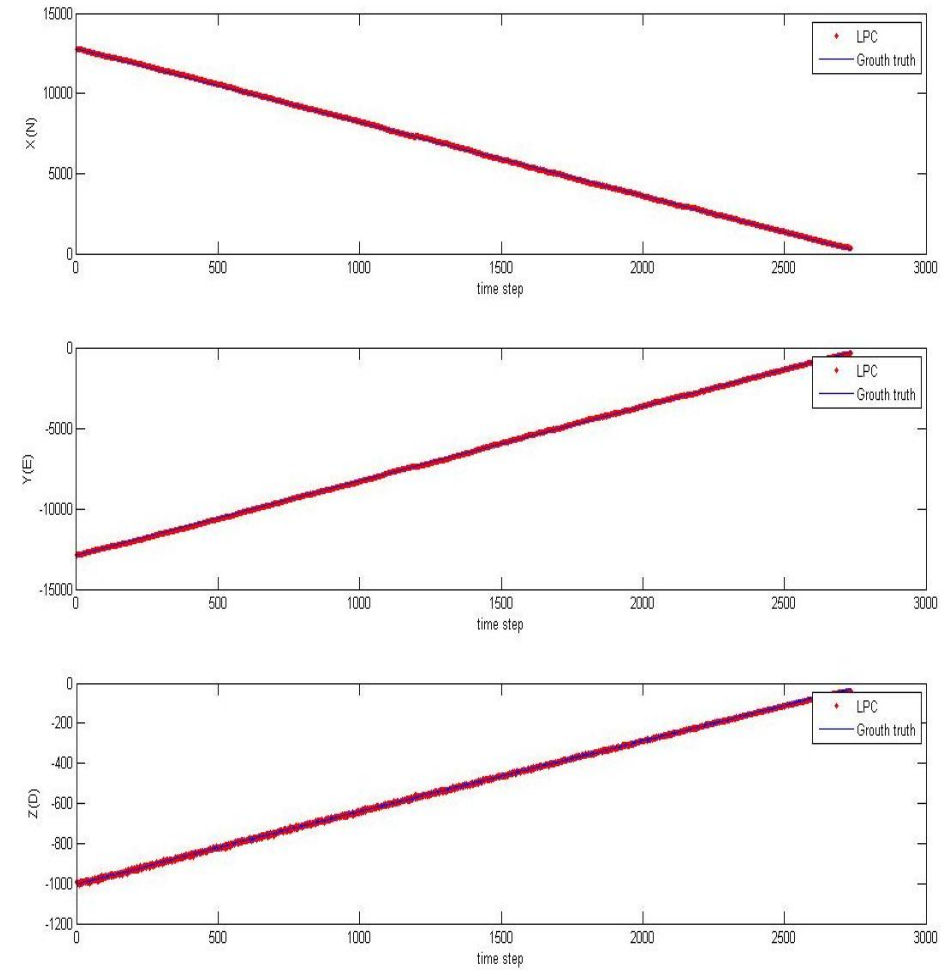

Fig. 7. Full trajectory estimation by particle filter in LPC

might be important for SLAM system such as unmanned aerial vehicles, autonomous underwater vehicles etc. Furthermore, it does not require additional computation resources. 

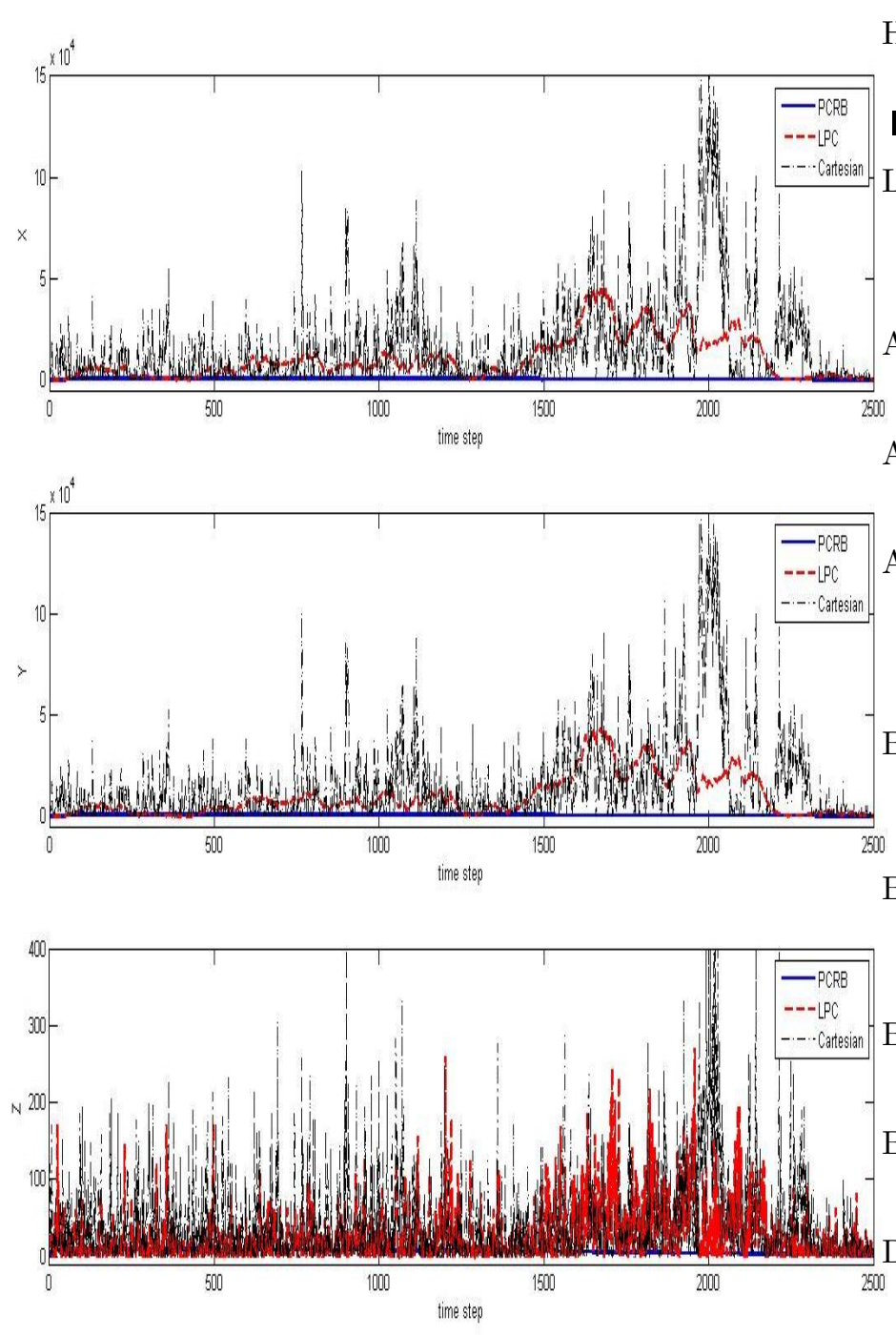

Fig. 8. Comparison of mean square error of particle filter in LPC and Cartesian

\section{ACKNOWLEDGEMENT}

This work was supported by the Pegase project.

\section{APPENDIX A}

Lemma 1. Given $F_{k-1}$ and $\mathcal{R}_{k-1}$, defined in equations (2) and (8), then

$$
F_{k-1}\left[I d_{2} \otimes \mathcal{R}_{k-1}\right]=\left[I d_{2} \otimes \mathcal{R}_{k-1}\right] F_{k-1} .
$$

Proof.

$$
\begin{aligned}
F_{k-1}\left[I d_{2} \otimes \mathcal{R}_{k-1}\right] & =\left[\left[\begin{array}{cc}
1 & \eta_{k-1} \\
0 & 1
\end{array}\right] \otimes I d_{3}\right]\left[I d_{2} \otimes \mathcal{R}_{k-1}\right] \\
& =\left[\begin{array}{cc}
1 & \eta_{k-1} \\
0 & 1
\end{array}\right] \otimes \mathcal{R}_{k-1}
\end{aligned}
$$

Moreover,

$$
\begin{aligned}
{\left[I d_{2} \otimes \mathcal{R}_{k-1}\right] F_{k-1} } & =\left[I d_{2} \otimes \mathcal{R}_{k-1}\right]\left[\left[\begin{array}{cc}
1 & \eta_{k-1} \\
0 & 1
\end{array}\right] \otimes I d_{3}\right] \\
& =\left[\begin{array}{cc}
1 & \eta_{k-1} \\
0 & 1
\end{array}\right] \otimes \mathcal{R}_{k-1}
\end{aligned}
$$

Hence,

$$
F_{k-1}\left[I d_{2} \otimes \mathcal{R}_{k-1}\right]=\left[I d_{2} \otimes \mathcal{R}_{k-1}\right] F_{k-1}
$$

Lemma 1 is applied for step 4 in equation (11).

\section{REFERENCES}

Aidala, V.J. and Hammel, S.E. (1983). Utilization of modified polar coordinates for bearings-only tracking. IEEE Trans. Automatic Control, 28(3).

Allen, R.R. and Blackman, S.S. (1991). Angle-only tracking with a MSC filter. In IEEE Conference on Digital Avionics Systems. USA.

Arulampalam, S., Maskell, S., Gordon, N., and Clapp, T. (2002). A tutorial on particle filters for on-line nonlinear/non-Gaussian Bayesian tracking. IEEE Trans. Signal Processing, Special Issue on Monte Carlo Methods, $174-188$.

Barat, C. and Lagadec, B. (2008). A corner tracker snake approach to segment irregular object shape in video image. In IEEE Conference on Acoustics, Speech, and Signal Processing. USA.

Brehard, T. and Le Cadre, J.P. (2004). A new approach for the bearings-only-problem: estimation of the varianceto-range ratio. In 7th International Conference on Information Fusion. Sweden.

Brehard, T. and Le Cadre, J.P. (2007). Hierarchical particle filter for bearings only tracking. IEEE Trans. Aerospace and Electronic Systems, 43(3).

Bryson, M. and Sukkarieh, S. (2005). Bearing-only SLAM for an airborne vehicle. In Australasian Conference on Robotics and Automation. Australia.

Dobrokhodov, V.N., Kaminer, I.I., Jones, K.D., and Ghabcheloo, R. (2006). Vision-based tracking and motion estimation for moving targets using small UAVs. In Proceedings of the American Control Conference. USA.

Hespanha, J.M., Yakimenko, O., Kaminer, I., and Pascoal, A. (2004). Linear parametrically varying systems with brief instabilities: an application to vision/inertial navigation. IEEE Trans. Aerospace and Electronic Systems, 40(3).

Kaminer, I., Kang, W., Yakimenko, O., and Pascoal, A. (2001). Application of nonlinear filtering to navigation system design using passive sensors. IEEE Trans. Aerospace and Electronic Systems, 37(1).

Kaminer, I., Pascoal, A., and Kang, W. (1999). Integrated vision/inertial navigation system design using nonlinear filtering. In Proceedings of the American Control Conference. USA.

Karlsson, R. and Gustafsson, F. (2001). Range estimation using angle-only target tracking with particle filters. In American Control Conference. USA.

Kolmogorov, V. and Zabih, R. (2004). What energy functions can be minimized via graph cuts? IEEE Trans. Pattern Analysis and Machine Intelligence, 26(2).

Stepanyan, V. and Hovakimyan, N. (2008). Visual tracking of a maneuvering target. Journal of Guidance, Control, and Dynamics, 31(1).

Tisdale, J., Ryan, A., Kim, Z., Tornqvist, D., and Hedrick, J.K. (2008). A multiple UAV system for vision-based search and localization. In IEEE American Controls Conference. USA. 